\title{
Quality Evaluation and Nonuniform Compression of Geometrically Distorted Images Using the Quadtree Distortion Map
}

\author{
Cristina Costa \\ Department of Information and Communication Technology (DIT), University of Trento, Via Sommarive 14, 38050 Trento, Italy \\ Email:costac@ing.unitn.it \\ Francesco G. B. De Natale \\ Department of Information and Communication Technology (DIT), University of Trento, Via Sommarive 14, 38050 Trento, Italy \\ Email: denatale@ing.unitn.it
}

Fabrizio Granelli

Department of Information and Communication Technology (DIT), University of Trento, Via Sommarive 14, 38050 Trento, Italy Email: granelli@ing.unitn.it

Received 1 September 2003; Revised 7 January 2004

\begin{abstract}
The paper presents an analysis of the effects of lossy compression algorithms applied to images affected by geometrical distortion. It will be shown that the encoding-decoding process results in a nonhomogeneous image degradation in the geometrically corrected image, due to the different amount of information associated to each pixel. A distortion measure named quadtree distortion map (QDM) able to quantify this aspect is proposed. Furthermore, QDM is exploited to achieve adaptive compression of geometrically distorted pictures, in order to ensure a uniform quality on the final image. Tests are performed using JPEG and JPEG2000 coding standards in order to quantitatively and qualitatively assess the performance of the proposed method.
\end{abstract}

Keywords and phrases: geometrical distortion, picture coding, quality evaluation.

\section{INTRODUCTION}

Images acquired by optical sensors usually present some kind of geometrical distortion due to the characteristics of lenses and sensors adopted in the acquisition system, or to the physical structure of the object under inspection, such as in the case of textures projected onto nonplanar surfaces [1]. In specific applications, such effects may also become more significant, due to the specific nature of the acquisition system. This is the case, for instance, of acquisition systems used in video surveillance or ambient intelligence applications, where wide-angle lenses are commonly used to acquire large areas with a single camera. In particular, fisheye lenses and panoramic lenses using omnidirectional mirrors are adopted to grab large portions of narrow indoor environments (a room, a car inside, etc.) $[2,3,4,5,6]$. Another application that strongly suffers from geometrical distortion is remote sensing [3].

In the projection of the real-world scene onto the image plane, the geometrical distortion acts as a nonlinear spatial compression and expansion of the luminance function in the pixel plane. This may cause problems in all the successive image treatment stages, from low-level processing to the interpretation of the scene, and can be partially solved by applying geometrical correction techniques based on sensor models and calibration processes. Unfortunately, the correction is only seldom operated at the sensor level, while it usually takes place at some remotely connected unit, where the application software is run. The geometrical correction may then happen to be carried out after the important processing steps have already been applied: in particular, compression and encoding of images is often implemented onboard to attain a more efficient transmission.

Some proposals to exploit the knowledge about the acquisition process to improve image processing have already been made, with application to specific domains such as medical teleradiology [7]. In [8], a generic and very simple acquisition model is studied, where the acquisition sensor is modelled through a modulation transfer function which simply introduces blurring. Another related work on the 


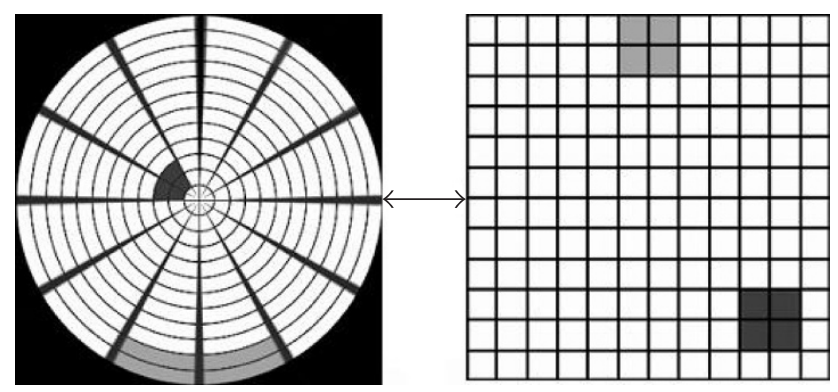

(a)

(b)

FIGURE 1: Graphical representation of a fisheye distorted image: (a) before and (b) after de-warping.

topic can also be found in [9], where the features of a retinalike sensor, associated with an omnidirectional mirror, are exploited for imaging purposes.

In this framework, we aim at investigating the impact of the geometrical distortion on image compression, and propose a new approach to improve the compression performance when encoding is applied to the geometrically distorted image. In our study, we will analyze both distorted images produced by real systems and synthetic images achieved by warping algorithms that simulate common distortion effects (fisheye and mirrored lenses). For this reason, we will use in the following the terms warping and distortion, as well as the opposite terms de-warping and geometric correction, referring to the same concepts.

The paper is organized as follows. In Section 2, the effects of nonlinear geometric distortions on co-decoded images are considered and the concept of quadtree distortion map (QDM) is introduced. In Section 3, QDM is used to design an adaptive image compressor able to achieve a uniform error distribution over the decompressed and de-warped image. It is also shown how this approach can be applied to the standard JPEG and JPEG2000 image compression algorithms, while maintaining full compliance only in the latter case. Finally, in Section 4, a selection of quantitative results is provided to demonstrate the viability and effectiveness of the proposed approach, and in Section 5, some conclusions are drawn.

\section{EVALUATION OF SPATIAL DISTORTION IMPACT IN IMAGE COMPRESSION AND QUADTREE DISTORTION MAP}

The first goal of this work is to evaluate the impact deriving from lossy co-decompression followed by geometric distortion correction on the final image quality.

The underlying assumption is that the image is compressed and decompressed before applying any geometrical correction. This hypothesis is reasonable in many practical systems due to several reasons, including necessity of ensuring low complexity of the acquisition system, use of sensors with embedded compression tools, frequent changes of optical lens or environment preventing the use of an embedded de-warping algorithm, and so forth. On the other hand, compression is increasingly used in the early stage of the acquisition, in particular for applications where the sensor is remotely connected to the processing unit using narrowbandwidth channels (e.g., wireless cameras) or is attached to a limited-capacity local storage device.

A spatial distortion in the acquisition system introduces a nonuniform distribution of the visual information in the acquired image. As a matter of fact, given two image areas with equivalent frequency content in the undistorted domain, the relevant areas in the acquired picture will show a higher frequency content where spatial compression occurred, and vice-versa. Conversely, the coding algorithm usually operates in a homogeneous way over the whole image. To achieve effective data compression, it must neglect some information, especially at the higher frequencies, and it has to produce an information loss as uniform as possible over the whole image, in order to avoid local peaks in the distortion.

Consequently, the error introduced by the encoder in an image region will be proportional to the local spatial deformation. Where spatial compression is present, the error will affect a larger zone in the final corrected image and will be more severe due to the presence of higher frequency contents. On the other side, in areas with low information density, the error will be attenuated by the averaging effect introduced by geometrical correction algorithms. Figure 1 depicts an example of this phenomenon related to the use of a fisheye lens, where the above concepts can easily find clear evidence. It can be observed that two areas of equal dimension in the undistorted (or corrected) domain, represented in dark and light grey in Figure 1b, are associated, in the distorted domain, to areas containing more or fewer samples according to their spatial position and to the geometry of the acquisition system.

In order to quantify this effect, the idea is to compare two schemes (see Figure 2): in the former, labelled as "scheme A," the acquired image is compressed and transmitted after the geometric correction; in the latter, "scheme B," compression and transmission are performed prior to the geometric correction of the image. The distortion is measured in any case by comparing the final result (decompressed, de-warped image) with the uncompressed, de-warped image, being the real-world (undistorted) picture unavailable in real cases.

A commonly accepted metric to estimate the distortion 


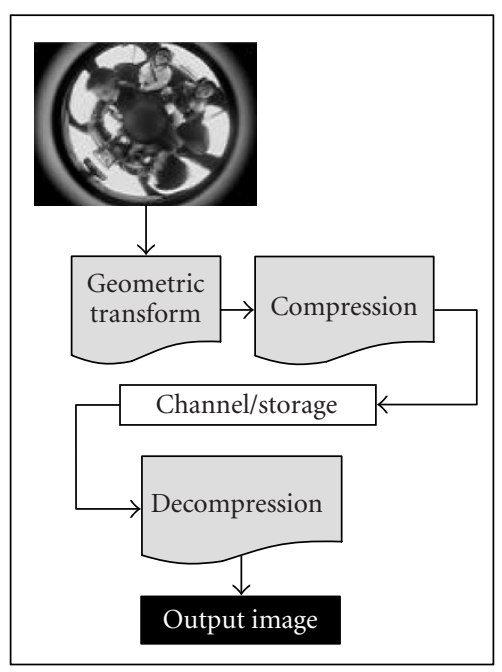

(a)

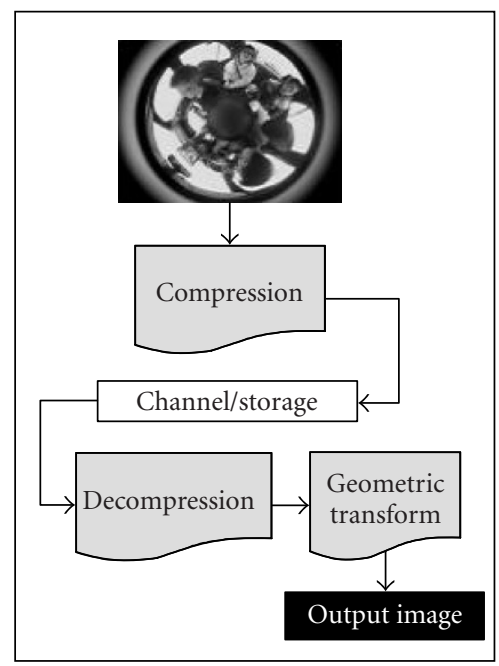

(b)

FIGURE 2: The two alternative compression and transmission schemes considered in the estimation of the impact of geometrical distortion on compression performance: (a) co-decoding is applied after geometrical correction, (b) vice-versa.

introduced by a processing system is the peak signal-to-noise ratio (PSNR), which treats the distortion as a kind of noise introduced on the original data, independently of its origin. The noise power is estimated through the computation of the mean square error (MSE), and the signal power is computed on the basis of the maximum excursion of the luminance function, namely,

$$
\operatorname{PSNR}(\mathrm{dB})=10 \log _{10} \frac{2^{2 b}}{\mathrm{MSE}},
$$

where $b$ is the number of bits per pixel (bpp) in the original image. Usually, PSNR is calculated on the whole image, but we are interested mostly in local measures that can highlight the nonhomogeneous distortion introduced by scheme $\mathrm{B}$ as compared to scheme A. For the purpose of evaluating the local distortion introduced by the process, we propose a method, called QDM, which uses a quadtree decomposition to generate a local map of the distortion effects. It will be demonstrated that QDM can be useful to evaluate the performance of compression schemes applied to geometrically distorted images, as well as to design optimized compression schemes able to improve the overall coding performance. It is to be pointed out that the concept of QDM is independent of the use of PSNR as a quality measure: QDM-based approaches can be implemented also using more sophisticated perceptual error models at the price of an increased complexity [10].

QDM is based on the application of the well-known quadtree decomposition algorithm [5]. The quadtree segmentation was demonstrated to efficiently represent simple image partitions subject to rigid geometric constraints. In our approach, the quadtree decomposition is applied to the error image $I_{\text {err }}(x, y)$, defined as the absolute difference performed on a pixel-by-pixel basis between the reference im- age $I_{\text {ref }}(x, y)$ (i.e., the geometrically corrected uncompressed image) and the output image (image after co-decompression and de-warping, in either order) $I_{\text {dist }}(x, y)$ :

$$
\forall(x, y), \quad I_{\mathrm{err}}(x, y)=\left|I_{\mathrm{ref}}(x, y)-I_{\text {dist }}(x, y)\right| .
$$

The aim is to obtain a map representing the spatial distribution of the distortion, through local measurements of the PSNR. The areas where PSNR is considered to be homogeneous are those identified by the leaves of the quadtree decomposition. The QDM algorithm is a recursive process and proceeds as follows.

(i) Compute the variance $\sigma^{2}$ of the error image $I_{\mathrm{err}}(x, y)$.

(ii) If $\sigma^{2}$ is greater than a given threshold $\Sigma$ th: then $\rightarrow$ split the image into four subimages, halving its size along $x$ and $y$ directions else $\rightarrow$ stop recursion.

(iii) Recursively apply steps (i) and (ii) to each subimage until each block fulfils the variance condition defined at point (ii) or reaches a minimum size $\Delta_{\min }$.

The stop condition in point (iii) takes into account also a minimum allowed dimension $\Delta_{\min }$ for each subimage, to avoid excessive splitting: in our tests, we used $\Delta_{\min }=8$, corresponding to the typical block size used in coding standards. $\Sigma$ th is set equal to $\alpha \cdot \sigma_{A}^{2}$, where $\sigma_{A}^{2}$ is the variance of the error image in Case $\mathrm{A}$, and $\alpha$ is a parameter in the range $1 \div 2$ taking into account the type of spatial distortion and the characteristics of the compression algorithm. In more detail, the choice of $\alpha$ is connected to the distortion introduced by the acquisition device, which largely depends on the viewing angle. For instance, the effect of fisheye lenses can be approximated by a spherical transform, in which the distortion is distributed over large image areas, while not reaching very high values. In this case, a low value of $\alpha$ (e.g., $1.2 \div 1.5$ ) 
TABLE 1: QDM statistics for JPEG encoder without adaptation, $C R=10,20$.

\begin{tabular}{|c|c|c|c|c|c|c|c|c|c|c|c|c|}
\hline & \multicolumn{6}{|c|}{$\mathrm{CR}=10$} & \multicolumn{6}{|c|}{$\mathrm{CR}=20$} \\
\hline & $\mathrm{PSNR}_{A}$ & $\mathrm{PSNR}_{B}$ & $\sigma_{A}^{2}$ & $\sigma_{B}^{2}$ & $\alpha$ & $\begin{array}{l}\text { No. of } \\
\text { generated } \\
\text { blocks }\end{array}$ & $\mathrm{PSNR}_{A}$ & $\mathrm{PSNR}_{B}$ & $\sigma_{A}^{2}$ & $\sigma_{B}^{2}$ & $\alpha$ & $\begin{array}{l}\text { No. of } \\
\text { generated } \\
\text { blocks }\end{array}$ \\
\hline $\begin{array}{l}\text { Tiled baboon } \\
\text { polar transform }\end{array}$ & 31.72 & 30.39 & 18.41 & 25.94 & 1.4 & 127 & 29.09 & 27.73 & 32.37 & 45.76 & 1.4 & 196 \\
\hline $\begin{array}{l}\text { Blood } \\
\text { polar transform }\end{array}$ & 33.56 & 30.60 & 12.61 & 29.61 & 1.4 & 208 & 27.17 & 23.78 & 62.10 & 129.16 & 1.4 & 184 \\
\hline $\begin{array}{l}\text { Tiled baboon } \\
\text { spherical mirror }\end{array}$ & 31.36 & 30.05 & 19.67 & 28.46 & 1.2 & 907 & 28.83 & 27.61 & 34.22 & 46.65 & 1.2 & 1483 \\
\hline $\begin{array}{l}\text { Blood } \\
\text { spherical mirror }\end{array}$ & 33.21 & 30.82 & 13.86 & 29.70 & 1.2 & 1333 & 29.89 & 27.34 & 30.20 & 66.41 & 1.2 & 1348 \\
\hline $\begin{array}{l}\text { Tiled baboon } \\
\text { parabolic mirror }\end{array}$ & 32.65 & 28.10 & 15.78 & 48.71 & 2.0 & 1396 & 29.78 & 26.01 & 28.75 & 72.74 & 2.0 & 1234 \\
\hline $\begin{array}{l}\text { Blood } \\
\text { parabolic mirror }\end{array}$ & 34.22 & 26.99 & 11.04 & 76.21 & 2.0 & 1786 & 30.80 & 24.68 & 23.82 & 122.23 & 2.0 & 1639 \\
\hline
\end{tabular}

TABLE 2: QDM statistics for JPEG2000 encoder without adaptation, CR = 10, 20.

\begin{tabular}{|c|c|c|c|c|c|c|c|c|c|c|c|c|}
\hline & \multicolumn{6}{|c|}{$\mathrm{CR}=10$} & \multicolumn{6}{|c|}{$\mathrm{CR}=20$} \\
\hline & $\mathrm{PSNR}_{A}$ & $\mathrm{PSNR}_{B}$ & $\sigma_{A}^{2}$ & $\sigma_{B}^{2}$ & $\alpha$ & $\begin{array}{l}\text { No. of } \\
\text { generated } \\
\text { blocks }\end{array}$ & $\mathrm{PSNR}_{A}$ & $\mathrm{PSNR}_{B}$ & $\sigma_{A}^{2}$ & $\sigma_{B}^{2}$ & $\alpha$ & $\begin{array}{l}\text { No. of } \\
\text { generated } \\
\text { blocks }\end{array}$ \\
\hline $\begin{array}{l}\text { Tiled baboon } \\
\text { polar transform }\end{array}$ & 29.73 & 27.19 & 38.32 & 61.84 & 1.6 & 172 & 27.05 & 24.50 & 62.90 & 103.47 & 1.6 & 169 \\
\hline $\begin{array}{l}\text { Blood } \\
\text { polar transform }\end{array}$ & 28.87 & 24.38 & 57.21 & 150.07 & 1.6 & 433 & 26.24 & 21.36 & 89.13 & 259.63 & 1.6 & 541 \\
\hline $\begin{array}{l}\text { Tiled baboon } \\
\text { spherical mirror }\end{array}$ & 29.36 & 26.74 & 38.04 & 69.36 & 1.5 & 730 & 27.47 & 24.47 & 53.13 & 104.35 & 1.5 & 1183 \\
\hline $\begin{array}{l}\text { Blood } \\
\text { spherical mirror }\end{array}$ & 28.51 & 25.08 & 60.95 & 136.11 & 1.5 & 967 & 26.46 & 22.57 & 86.10 & 207.95 & 1.5 & 1399 \\
\hline $\begin{array}{l}\text { Tiled baboon } \\
\text { parabolic mirror }\end{array}$ & 30.84 & 25.59 & 29.87 & 88.08 & 2.0 & 1330 & 27.05 & 24.50 & 62.90 & 103.47 & 1.6 & 169 \\
\hline $\begin{array}{l}\text { Blood } \\
\text { parabolic mirror }\end{array}$ & 30.22 & 22.98 & 42.33 & 207.23 & 2.0 & 1549 & 26.24 & 21.36 & 89.13 & 259.63 & 1.6 & 541 \\
\hline
\end{tabular}

is required to achieve a precise QDM map. On the other side, parabolic or conic projections typical of mirrored lenses produce heavier distortions, thus requiring higher values of $\alpha(1.6 \div 2)$ to focus on greatly distorted areas. Consequently, it has been found that it is possible to heuristically set $\alpha$ a priori on the basis of the type of geometrical distortion, independently of the image content. Further considerations on the setting of $\alpha$ are provided in Section 4 (Tables 1 and 2 and relevant discussion), where the impact of the coding algorithm is also considered. 


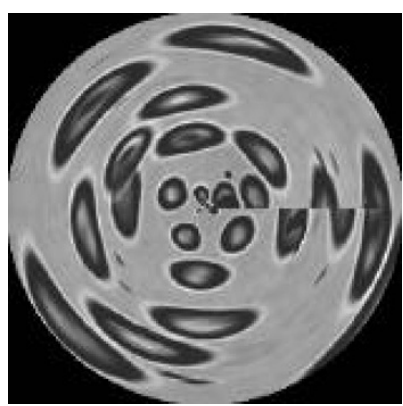

(a)

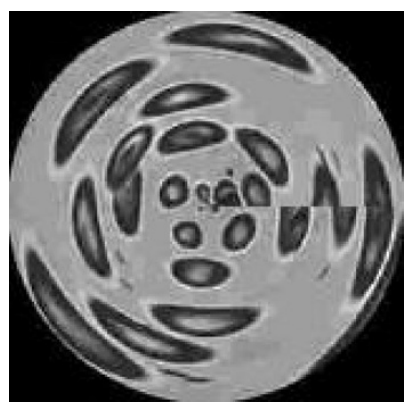

(b)

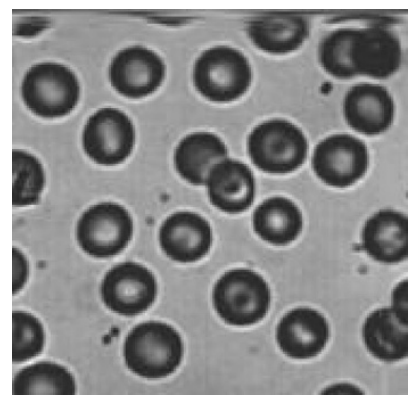

(c)

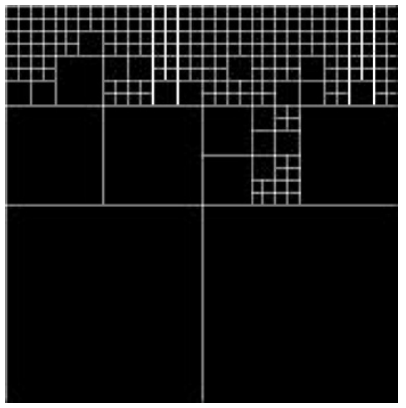

(f)

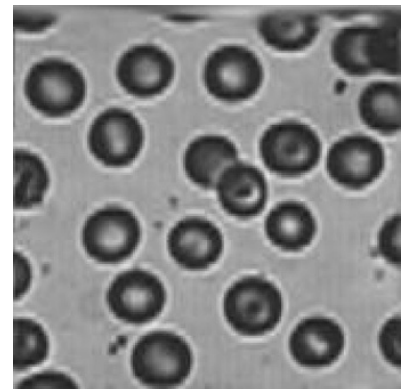

(d)

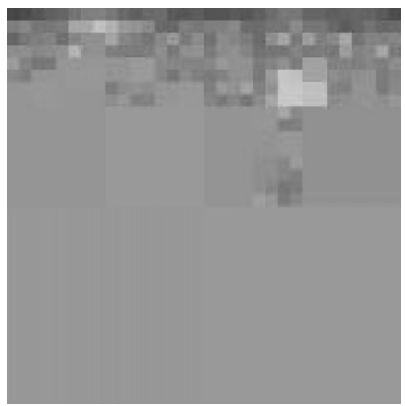

(g)

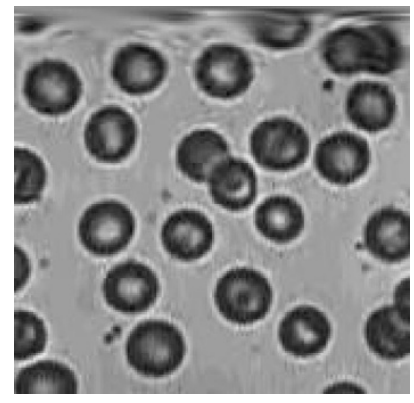

(e)

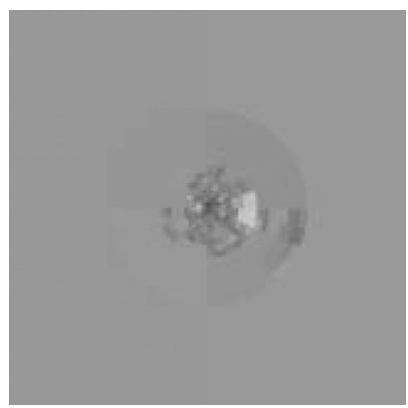

(h)

FIGURE 3: Example of application of QDM: (a) original, uncompressed and warped by polar transform, (b) compressed in warped domain, (c) original, uncompressed de-warped, (d) output of scheme A, (e) output of scheme B, (f) result of split process scheme B, (g) QDM map scheme B, (h) polar transform of QDM map scheme B. Note that when the split process is applied to scheme A (with the same parameters used for scheme B), there is no split at all and the QDM map is a constant value image.

As a consequence of the above procedure, the areas are split where the error is more fluctuant, thus achieving a subdivision of the error image into areas with nearly constant distortion. The result of the decomposition is a sparse matrix that indicates the block subdivision of the error image in blocks of various dimensions, associated to different error values. In Figure 3, an example of QDM is shown with application to the "blood" test image, $256 \times 256,8 \mathrm{bpp}$. Here, the distortion introduced by the acquisition system is simulated by a polar coordinate transform in Figure 3a, which reproduces the behaviour of a $360^{\circ}$ mirrored lens. The error is computed between the reference image (uncompressed de-warped) in Figure 3c and the outputs of schemes "A" and "B," in Figures 3d and 3e, respectively. A standard JPEG en- coder with compression ratio $(\mathrm{CR})=10$ was used in both cases (the co-decoded images in warped and de-warped domains are shown in Figures $3 \mathrm{~b}$ and $3 \mathrm{~d}$, respectively), while the parameter $\alpha$ was set to 1.4 . The CR is defined as

$$
\mathrm{CR}=\frac{N_{b, o}}{N_{b, c}},
$$

where $N_{b, o}$ is the number of bits required for representing the original image in the canonical form and $N_{b, c}$ the number of bits after compression.

Since the variance threshold $\Sigma$ th is higher than the error variance in scheme $\mathrm{A}$, the relevant output image does not produce any split. As far as scheme B is concerned, the 
result of the splitting process is represented in Figure 3f. In Figure 3g, called QDM map, each leaf of the relevant quadtree is associated to a grey level proportional to the local distortion (the higher the distortion, the darker the corresponding block). The QDM map of scheme B makes evident that the compression in the distorted domain generates an uneven distribution of the error. To better appreciate this fact, in Figure $3 \mathrm{~h}$ the QDM map associated to scheme B is transformed back in polar coordinates, that is, in the original acquisition domain. The resulting map provides a convincing confirmation of the above reasoning about the implications of lossy compression applied to geometrically distorted images. As a matter of fact, it can be observed that the quality degradation progressively increases towards the image centre, where the information density is higher (due to spatial compression).

It is important to point out that in the compression of natural images, the distribution of the error can fluctuate also in the absence of geometrical distortions, due to the nonstationarity of the input image and the characteristics and parameters of the encoder. Nevertheless, this effect can be neglected for two reasons.

First, the image content is the same for both scheme A and $\mathrm{B}$, thus allowing a comparative assessment. The underlying assumption is that the effects of nonstationary image contents and geometrical distortions on the error distribution are uncorrelated and additive. This is not completely true in general, due to the fact that a geometrical deformation can alter not only the magnitude but also the orientation of spatial frequencies (e.g., straight lines become curves when acquired by a wide-angle lens). Therefore, due to the different treatment of the spatial frequencies at the encoder, the distortion can have some "second-order" effects on the final result. Nevertheless, these phenomena are related more to the perceptive quality of the decompressed image than to its objective assessment, and therefore can be neglected in QDM, which is simply based on absolute error estimation.

Second and more important, in practical applications QDM is meant to be performed off-line, by presenting to the system some predefined calibration images, designed to match the application to which the acquisition system is targeted. For instance, in a fixed camera surveillance system, the calibration set could be obtained by selecting some shots acquired in typical operating conditions, thus allowing to take into account also the local image content. On the contrary, to achieve a general purpose system, the calibration image should have a frequency content as uniform as possible, to ensure a uniform behaviour independently of the application. According to this last model, in our tests we used images containing statistical or structural textures, as in the case of the blood image, or synthetic patterns obtained by patch repetition.

A further consideration about system calibration concerns the possibility of computing the distortion map a priori, simply based on the characteristics of the acquisition system. For instance, it would be possible to determine the local compression and expansion due to the geometrical deformation, and directly estimate the relevant impact on the com- pression distortion. Unfortunately, this is not a trivial task, since the deformation produces in general a resampling of the picture over an irregular sampling grid, which in turn generates very different spatial frequencies (both in magnitude and in orientation). Moreover, such spurious frequencies can be treated differently by different compression tools, thus having various impacts on the final quality. Therefore, although a simple "pixel density map" would probably cope with a local resampling over a regular grid, more complex deformations would require sophisticated warping models, as well as a specific customization whenever the acquisition system and/or the compression tool is changed. On the contrary, the proposed method shows the advantage of a very simple calibration procedure to be performed with a standard procedure.

\section{ADAPTIVE COMPRESSION OF GEOMETRICALLY DISTORTED IMAGES USING QDM}

Previous sections demonstrated that the application of lossy compression to a geometrically distorted image will produce areas that are more heavily damaged with respect to others. The observation of the QDM map well identifies the presence and the extent of this problem. In this section, we explore the possibility of exploiting the QDM to perform a nonuniform compression of the distorted image, in order to compensate this problem. This will be achieved by allocating the bits in a more efficient way, thus providing both higher compression efficiency and better quality of reconstruction. As a rule of thumb, the aim is to allocate more bits to the areas that are more sensitive to distortion, in order to represent them with a quality comparable to other image regions.

The concept of optimal bit allocation is not new in itself. Several compression schemes, either based on DCT or wavelet transforms, adopt bit allocation algorithms that aim at optimizing the rate-distortion function or some objective or subjective local quality measures (see [11] and references therein). Although these algorithms are able to take advantage of the local characteristics of the image to be compressed, none of them was explicitly designed to include external factors that may affect the importance of some bits with respect to others. In the problem under consideration, we determine the influence of geometric distortions on the coded image and use this information to achieve a geometric-distortion-driven bit allocation. It is to be pointed out that such a scheme may be integrated with different ratedistortion optimization strategies, provided that the QDM parameters are taken into account as additional input for the bit allocation process. QDM determines in fact a map that allows data prioritization to be usable in combination with other techniques. In this sense, an important advantage of QDM-based compression is the possibility to comply with different standard compression algorithms with region of interest (RoI) support for implementing geometric distortion compensated compression.

A conceptual architecture of the proposed approach is presented in Figure 4 (scheme "C"). Referring to a such scheme, the core methodology is in the RoI-based compres- 


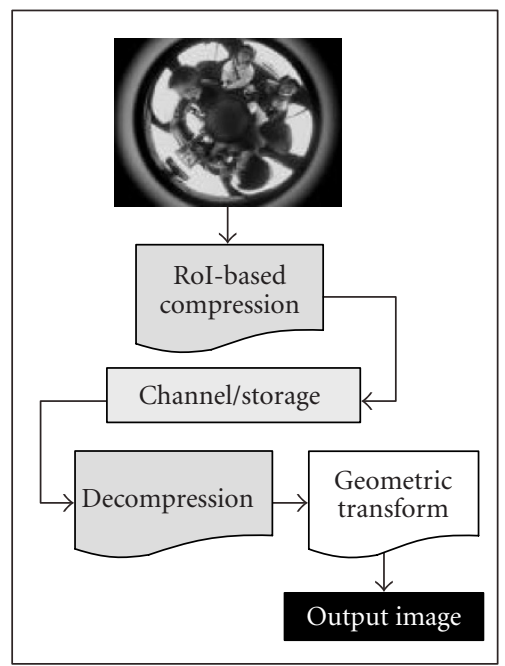

FIGURE 4: Conceptual scheme of the proposed approach.

sion block, which is responsible for the adaptive allocation of the bits to different image areas. The adaptation process is driven by the computation of the QDM map, which is performed off-line using a suitable image or set of images to calibrate the system. In the following subsections, we will present two different approaches, in which the scheme " $\mathrm{C}$ " is inserted within JPEG and JPEG2000 standard codecs, respectively. Is it to be pointed out that only in the case of JPEG2000 is it possible to ensure full compliance, while JPEG requires the use of additional information not included in the standard syntax.

\subsection{Adaptive compression using a JPEG-like scheme and QDM}

As a first trial of the above concepts, the adaptive scheme " $\mathrm{C}$ " was applied to a JPEG compression algorithm [4]. In JPEG compression, the image is divided into $8 \times 8$ blocks, each block is DCT-transformed, and the relevant coefficients are quantized according to a predefined matrix, scanned in zigzag order, associated to opportune codewords, and entropy coded. The key operation to achieve effective compression is the coefficient quantization, which is also responsible for the distortion of the reconstructed image.

The degree of compression and the relevant quality are tuned by a single parameter $Q$, which determines the quantization step. In standard JPEG, the value of the parameter $Q$ is kept constant across the whole image, making impossible a spatial adaptation of the coding scheme. In our analysis, we modified this aspect by considering the possibility of changing the parameter $Q$ in different image areas. This generates a noncompliant compression tool, which was studied only for the purpose of analysis and comparison.

We suggest using different values of $Q$ that can be alternatively selected in the encoding of each block. By selecting the appropriate factor, it is possible to assign more bits to blocks in the areas more sensitive to the coding error by modulating the quantization step used for the relevant coefficients. The choice is performed on a block-by-block basis taking into account the QDM map. For the sake of simplicity, in our tests we considered a binary classification of the image blocks associated to two quantization parameters, the lower of which is associated to a region of interest. In this case, the information necessary to the decoder to appropriately set the $Q$ factor in the de-quantization process can be transmitted as an additional bit per block, as a map for the whole image, or should be directly reconstructed at the receiver without any additional overhead, provided that the QDM map is available at the decoder. The overhead deriving from the first solutions is fixed to 0.015 bit per pel ( 1 bit for each $8 \times 8$ block), while being much lower in the second approach, which allows one to exploit the spatial correlation of the QDM and/or efficient quadtree encoding techniques (see [12] for an example). In either cases, the overhead is low enough to be considered negligible.

\subsection{Adaptive compression using JPEG2000 and QDM}

Far more interesting is the use of QDM as a tool to automatically define an RoI in the framework of the JPEG2000 still picture coding standard [13]. In fact, although JPEG2000 defines the format and profiles of the compressed bitstream, it leaves higher freedom on the parameter setting, and includes a specific syntax to set an RoI within an image, to which a higher priority and quality can be assigned. A further advantage of JPEG2000 is that the compressed image can be truncated at any point and still be correctly decoded. If the RoI mechanism is used, the information related to the specified area will get a higher priority in the bitstream and will be preserved in the case of transport problems. This characteristic can be used in conjunction with transmission protocols such as JPIP $[14,15]$ to allow progressive transmission and decoding of the image.

In JPEG2000, the subband information is divided into several code blocks. The bit allocation associated to each of these code blocks is determined by a rate-distortion optimization process. In the case of SNR scalable coding, different "quality" layers are created to accommodate different rates. The different rate-distortion pairs are defined by an algorithm, the postcompression rate-distortion (PCRD), applied after the compression of the image, and have the property of satisfying the optimal rate-distortion curve. Bitstream truncation at rates in between those indicated leads to a suboptimal solution, whose precision depends on the number of quality layers.

There are two different mechanisms in JPEG2000 for implementing RoI encoding: the max-shift method and the "implicit" RoI encoding. In implicit RoI encoding, the encoder uses RoI information for modulating the distortion cost function that drives the PCRD algorithm responsible for the code-block bit allocation. A detailed description of the implicit RoI encoding is given in [16]. The implicit RoI encoding was chosen for implementing the nonuniform compression with QDM since it allows a smooth transition among regions. 


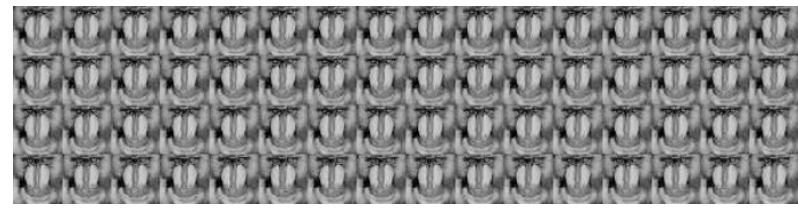

(a)

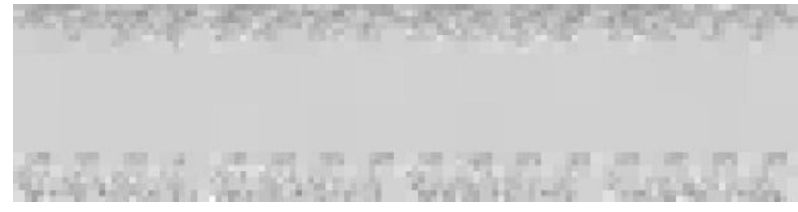

(b)

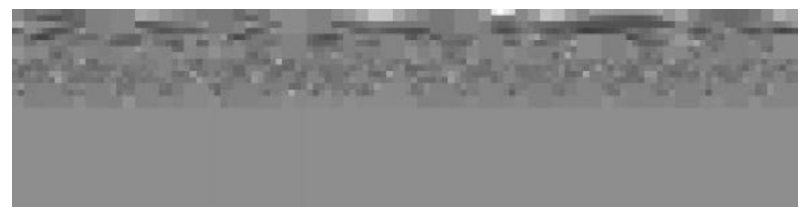

(c)

FIGURE 5: QDM maps of a test image: (a) original achieved by patch repetition of baboon image; (b) QDM map for semispherical mirror; (c) parabolic mirror.

According to scheme " $\mathrm{C}$," the RoI is determined from the analysis of the QDM map and is directly implemented within the standard, thus ensuring full compliance. Future investigations will address the possibility of defining scalability at a finer granularity, for example, directly adapting the distortion cost function with the information derived from the QDM. With this approach, it is possible to fully to take advantage of information gathered with the QDM algorithm instead of using a binary mask for RoI definition.

\section{EXPERIMENTAL RESULTS}

\subsection{Quality measurement}

QDM was applied to images distorted by different geometric transforms. A basic rectangular-to-polar coordinate transform was initially used to assess the sensitivity of the QDM measure [17]. To create the QDM map for polar distortion, a test set was built containing images with almost uniform frequency content (see blood image, Figure 4), as well as images artificially generated by tiling a patch taken from a natural image. Additionally, tests were performed on distortions derived from panoramic mirrors, typical of omnidirectional vision systems. In Figure 5, the QDM maps are shown, relevant to semispherical mirror and parabolic mirror transforms (Figures $5 b$ and $5 c$, respectively) of image in Figure $5 a$ (patch replication of the image baboon).
As far as the compression is concerned, both JPEG and JPEG2000 profiles at medium to high compression ratios are used. In Tables 1 and 2, the measures of total PSNR $A, B$ and $\sigma_{A, B}^{2}$ are reported for JPEG and JPEG2000 compression, respectively, at two different CRs (10 and 20, respectively). It is to be observed that scheme A always performs better than scheme B, from the viewpoint of both objective quality and homogeneous distribution of the distortion (lower error variance). The tables include also the values assigned to the $\alpha$ parameter and the relevant number of generated leaves in the quadtree. From the analysis of these data, it is possible to note that acquisition systems associated to a higher distortion (e.g., the parabolic mirror) generate a more complex quadtree even for higher values of $\Sigma$ th, thus being associated to higher values of $\alpha$.

\subsection{Nonuniform compression using JPEG}

In this case, we based our considerations on a modified JPEG compression scheme. In particular, a rectangular QDMbased RoI is identified that encompasses the image area affected by higher geometrical deformation. For instance, Figure 6 refers to a polar transformation of the blood image. The area containing the higher QDM values is bounded by a white rectangle in the original image domain (Figure 6a) and in the QDM map (Figure 6b). During compression, a higher amount of bits is allocated to the blocks falling within the RoI. This configuration is labelled in the results as case "C." In the chart of Figure 7, quality measurements related to this test are reported and compared. In our tests, the quality parameters used to encode the background and the RoI were set to $Q$ and $Q+10$, respectively.

\subsection{Nonuniform compression using JPEG2000}

Finally, we used the QDM to derive a mask for RoI encoding using the JPEG2000 architecture. The images are compressed in a progressive lossy to lossless JPEG2000 codestream using the Kakadu JPEG2000 codec software [18], with the lower layer encoded with a CR equal to 400. In the QDM compression scheme, an implicit ROI is implemented using a mask image derived from the QDM map as an input to the JPEG2000 encoder. A reversible compression with a progressive lossy to lossless code-stream having 3 layers is applied to the image. The parameter R-weight, that controls transition from background to foreground, is set to 4, and the parameter R-level is set to 3. Different compression rates can be achieved decoding only the initial portion of the code-stream, corresponding to the overall desired bit rate.

The plot in Figure 8 summarizes the results achieved in terms of PSNR, which underlines the advantages of the QDM-driven encoder (scheme "C"). Visual results are presented in Figures 9 and 10 for an average compression value $(\mathrm{CR}=20)$.

Finally, some examples of off-line image system calibration are provided in Figures 11 and 12. Figure 11 reports the result obtained by compressing a frame of the mobile and calendar images sequence using a QDM map generated 


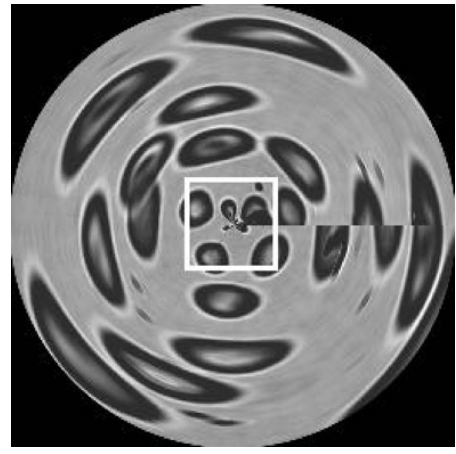

(a)

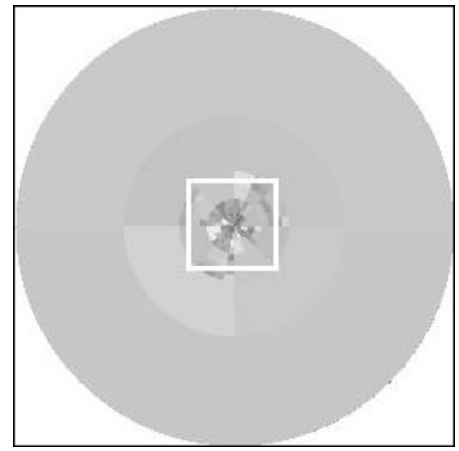

(b)

FIGURE 6: Identification of an RoI from the QDM of a distorted image: (a) image blood distorted by a polar transform; (b) the relevant QDM map and RoI (in white).

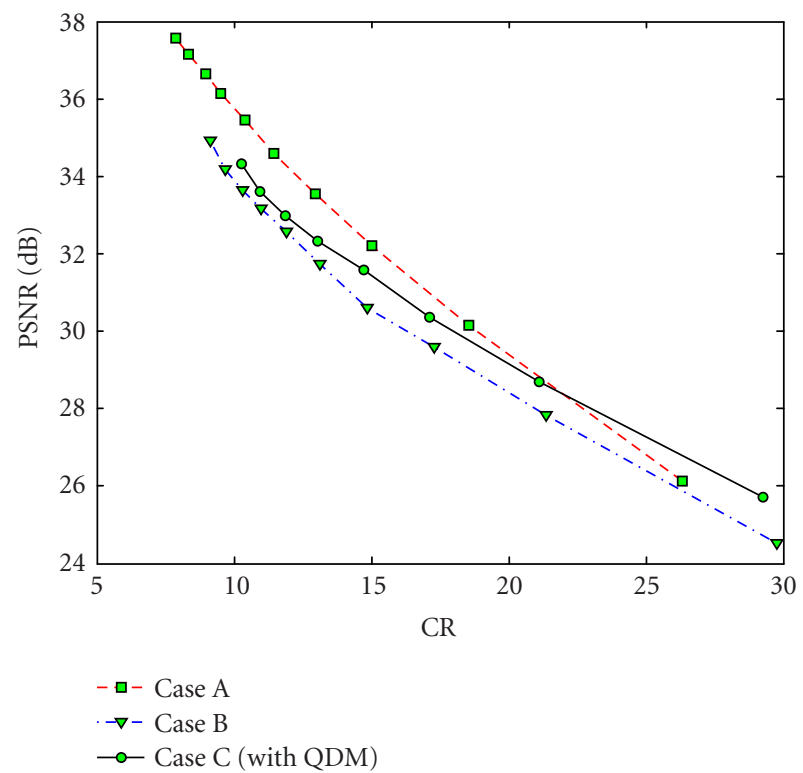

Figure 7: Comparison between compression schemes "A," "B," and "C," at increasing CR (blood image, polar transform, JPEG-like encoder).

off-line using a calibration pattern (the tiled baboon in Figure 10a). In Figure 12a, a synthetic scene created with the ray tracing software PovRay (Persistence of Vision Raytracer, http://www.povray.org) was used to simulate an omnidirectional vision system based on a spherical mirror. A previously generated QDM map using a calibration image was used for compression. Comparing the images generated in Case B (Figures 12b and 12c) and in Case C (Figures 12d and $12 \mathrm{e}$ ), it is possible to appreciate the perceptual quality improvement introduced by the QDM compression algorithm.

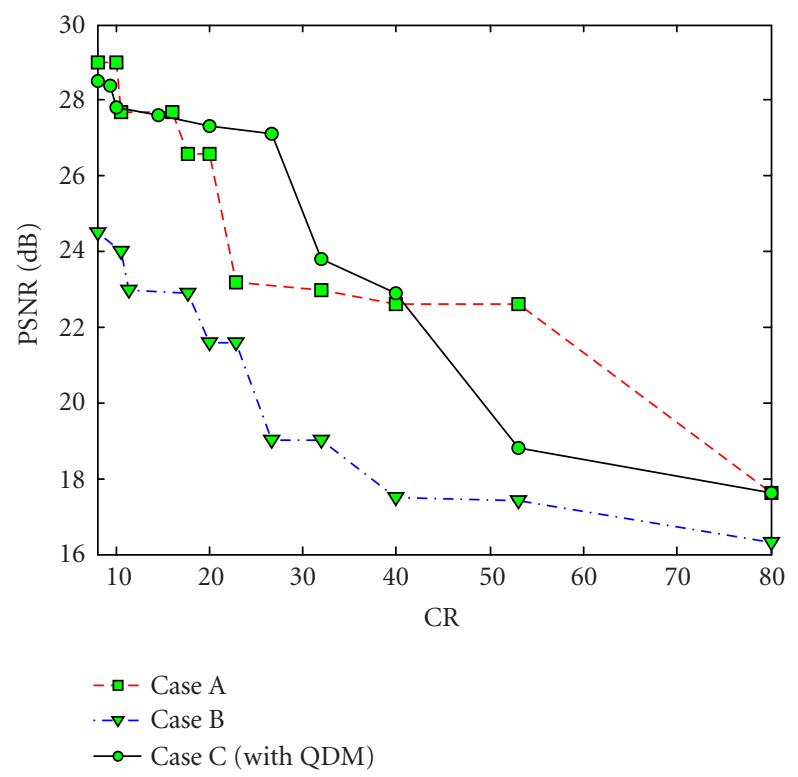

Figure 8: Comparison between compression schemes "A," "B," and "C," at increasing CR (blood image, polar transform, JPEG2000 encoder).

\section{CONCLUSIONS AND FUTURE WORK}

A novel method called quadtree distortion map (QDM) for the measurement of the compression error due to geometrical image distortion is presented. QDM provides a useful tool to quantitatively assess the effects of spatial deformations in image coding. Furthermore, it can be used to design adaptive nonuniform compression schemes based on standard encoding techniques such as JPEG and JPEG2000.

Experimental results underline that the proposed metric is effective in defining the regions of distorted images that are more sensitive to loss of information introduced by com- 


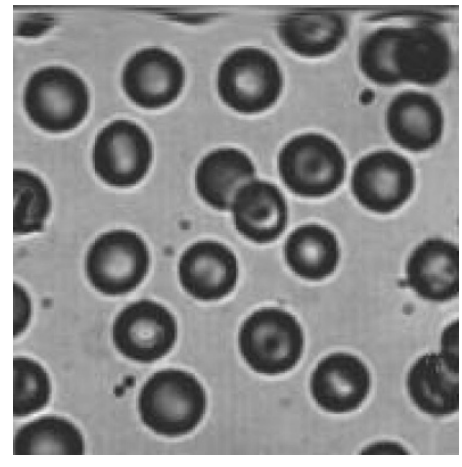

(a)

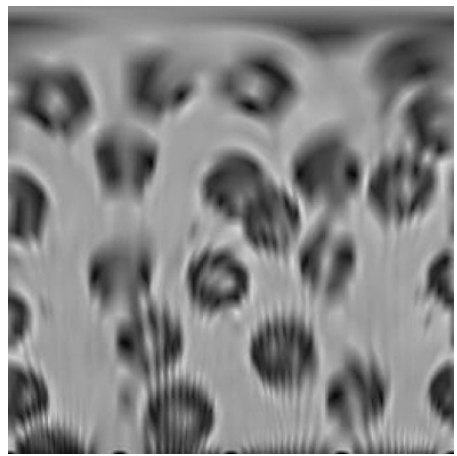

(c)

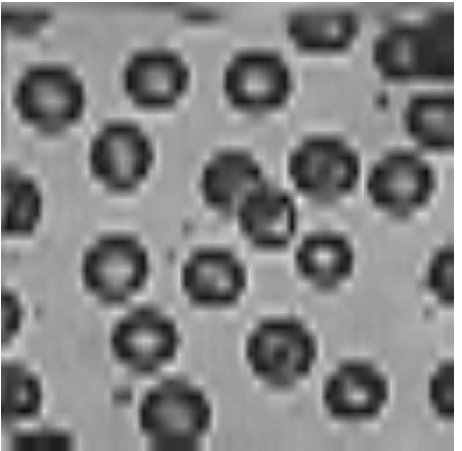

(b)

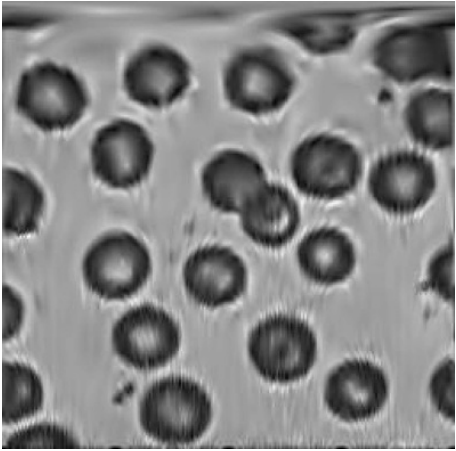

(d)

FIGURE 9: Performance comparison of schemes “A," “B”, and “C”" on blood image at CR = 20: (a) reference image; (b) JPEG2000 compression after geometric transform (scheme "A"); (c) JPEG2000 compression before geometric transform (scheme "B"); (d) JPEG2000 QDM-driven nonuniform compression before geometric transform (scheme " $\mathrm{C}$ ").

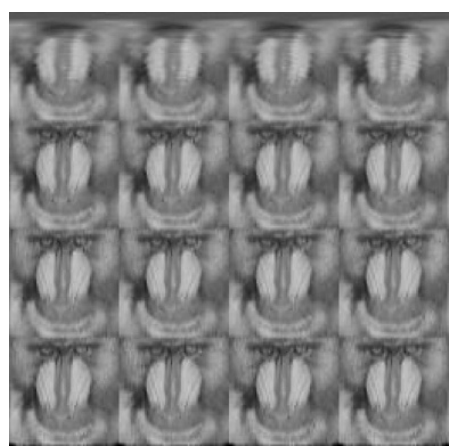

(a)

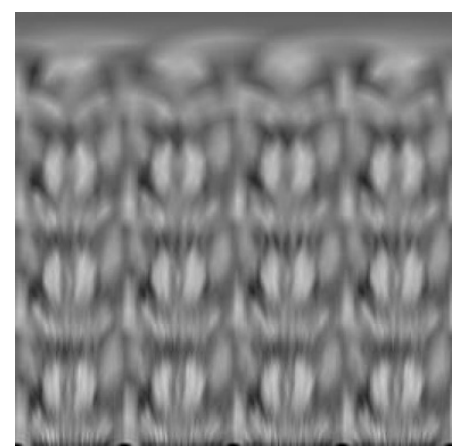

(b)

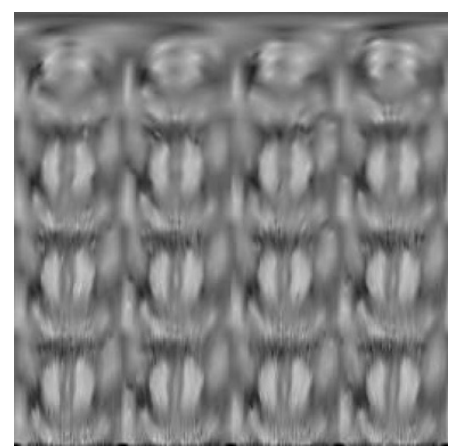

(c)

FIGURE 10: Performance comparison of schemes "B" and "C" on tiled baboon image at CR = 20: (a) reference image; (b) JPEG2000 compression before geometric transform (scheme "B”); (c) JPEG2000 QDM-driven nonuniform compression before geometric transform (scheme "C").

pression and thus it provides a useful support for encoder optimization in presence of visual distortions.

Further research will allow extending the model to other distortions and will test the proposed scheme in the framework of video coding, where estimation of the distortion should be supported by temporal redundancy of the source. 


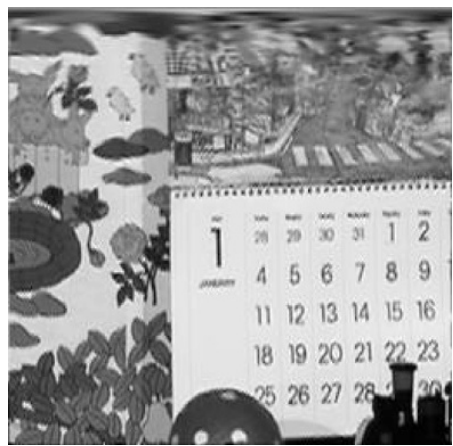

(a)

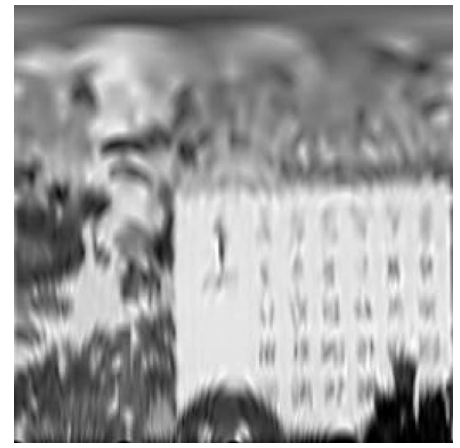

(b)

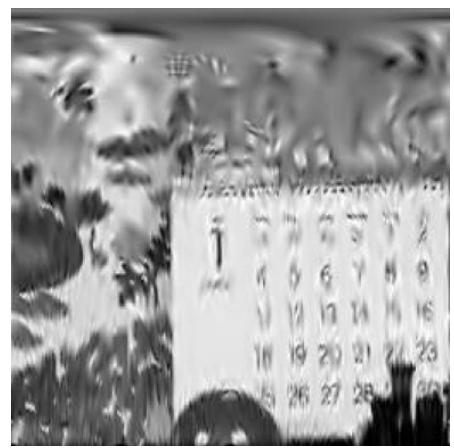

(c)

FIGURE 11: Performance comparison of schemes "B" and "C" on a frame of mobile and calendar sequence (details) at CR = 20, using off-line QDM calibration: (a) reference image; (b) JPEG2000 compression before geometric transform (scheme "B”); (c) JPEG2000 QDM-driven nonuniform compression before geometric transform (scheme "C").

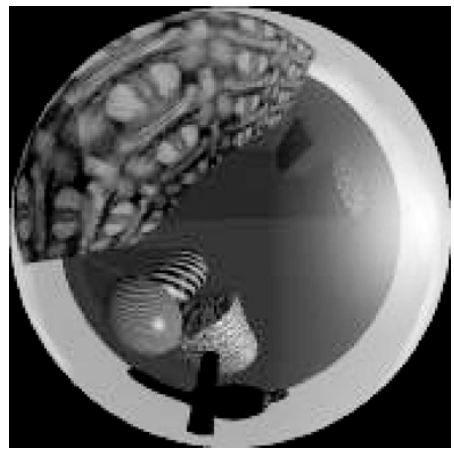

(a)

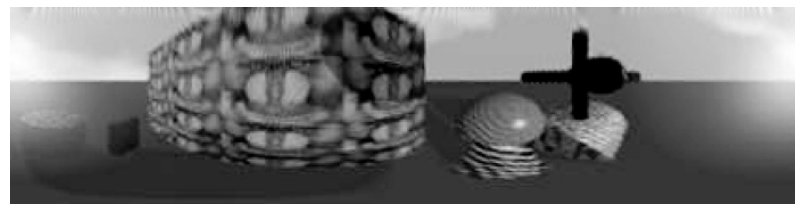

(b)

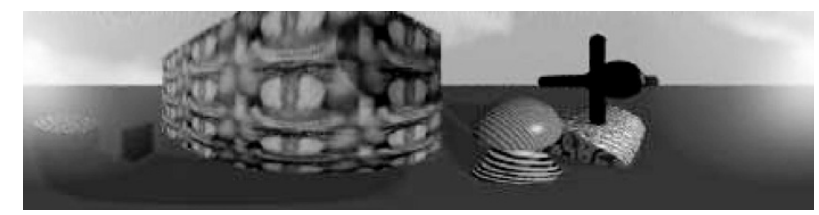

(d)

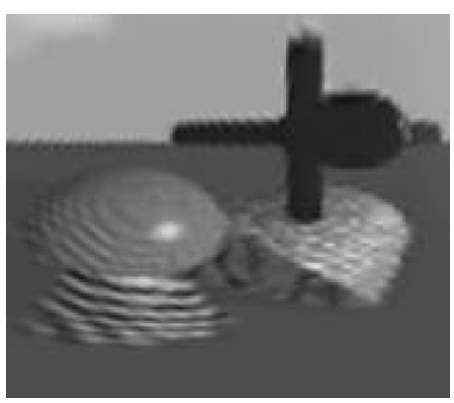

(c)

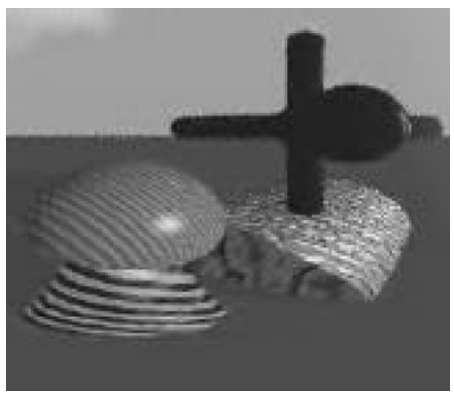

(e)

FIGURE 12: Performance comparison of schemes "B" and "C" on a synthetic image generated by PovRay at CR = 20, using off-line QDM calibration: (a) original; (b) JPEG2000 compression before geometric transform (scheme "B”); (c) details; (d) JPEG2000 QDM-driven nonuniform compression before geometric transform (scheme "C"); (e) details. 


\section{ACKNOWLEDGMENT}

This work was partially supported by the European Union within the framework of the IST Programme (VFP), AIDER research project (IST-2000-28058).

\section{REFERENCES}

[1] H. Farid, "Reconstructing ancient Egyptian tombs," in Proc. International Symposium on Virtual and Augmented Architecture, Dublin, Ireland, 2001.

[2] H. S. Sawhney and R. Kumar, "True multi-image alignment and its application to mosaicing and lens distortion correction," IEEE Trans. on Pattern Analysis and Machine Intelligence, vol. 21, no. 3, pp. 235-243, 1999.

[3] A. M. Wu and Y. Y. Lee, "Geometric correction of high resolution image using ground control points," in Proc. Asian Conference on Remote Sensing (ACRS '01), vol. 2, pp. 1165-1168, Singapore, November 2001.

[4] G. K. Wallace, "The JPEG still picture compression standard," Communications of the ACM, vol. 34, no. 4, pp. 31-44, 1991.

[5] H. Samet and R. E. Webber, "On encoding boundaries with quadtrees," IEEE Trans. on Pattern Analysis and Machine Intelligence, vol. 6, no. 3, pp. 365-369, 1984.

[6] S. K. Nayar, "Omnidirectional vision," in Proc. 8th International Symposium on Robotics Research (ISRR '97), Shonan, Japan, October 1997.

[7] R. M. Cramblitt and K. J. Parker, "Ultrasound image compression exploiting image formation models," in Proc. IEEE Ultrasonics Symposium, vol. 2, pp. 1377-1380, San Antonio, Tex, USA, November 1996.

[8] C. Parisot, M. Antonini, M. Barlaud, S. Tramini, C. Latry, and C. Lambert-Nebout, "Optimization of the joint coding/decoding structure," in Proc. IEEE International Conference on Image Processing (ICIP '01), vol. 3, pp. 470-473, Thessaloniki, Greece, October 2001.

[9] G. Sandini, J. Santos-Victor, T. Pajdla, and F. Berton, "OMNIVIEWS: direct omnidirectional imaging based on a retinalike sensor," in Proc. 1st IEEE International Conference on Sensors (IEEE Sensors '02), pp. 27-30, Orlando, Fla, USA, June 2002.

[10] D. Tompa, J. Morton, and E. Jernigan, "Perceptually based image comparison," in Proc. International Conference on Image Processing (ICIP '00), vol. 1, pp. 489-492, Vancouver, BC, Canada, September 2000.

[11] A. Ortega and K. Ramchandran, "Rate-distortion methods for image and video compression," IEEE Signal Processing Magazine, vol. 15, no. 6, pp. 23-50, 1998.

[12] M. Accame and F. Granelli, "Hierarchical progressive image coding controlled by a region based approach," IEEE Transactions on Consumer Electronics, vol. 45, no. 1, pp. 13-20, 1999.

[13] C. Christopoulos, A. Skodras, and T. Ebrahimi, "The JPEG2000 still image coding system: an overview," IEEE Transactions on Consumer Electronics, vol. 46, no. 4, pp. 1103$1127,2000$.

[14] D. Taubman and R. Prandolini, "Architecture, philosophy, and performance of JPIP: internet protocol standard for JPEG2000," in Proc. International Symposium on Visual Communications and Image Processing (VCIP '03), vol. 5150 of Proceedings of SPIE, pp. 791-805, Lugano, Switzerland, July 2003.

[15] ISO/IEC JTC 1/SC 29/WG 1 N2904, "Information tech-
nology_JPEG 2000 image coding system-Part 9: Interactivity tools, APIs and protocols (JPIP)," 1st Committee Draft, Seoul, South Korea, March 2003.

[16] D. Taubman and M. Marcellin, JPEG2000: Image Compression Fundamentals, Standards and Practice, Kluwer Academic, Boston, Mass, USA, 2002.

[17] C. Costa, F. G. B. De Natale, and F. Granelli, "Quality evaluation and non-uniform compression of geometrically deformed images using the Quadtree Distortion Map (QDM)," in IEEE-Eurasip Workshop on Nonlinear Signal and Image Processing (NSIP '03), Grado, Italy, June 2003.

[18] Kakadu Software, Implementation of the JPEG2000 standard, Part 1 (ISO/IEC 15444-1), http://www.kakadusoftware.com/.

Cristina Costa was born in Santos, Brazil, in 1971. She received the Laurea (M.S.) degree in electronic engineering from the University of Genoa, Italy, in 1996, with a thesis on image registration. As an exchange student, she worked at the School of Electronic Engineering, Information Technology and Mathematics, Surrey University, UK, developing algorithms for image-processing applications. She continued her studies receiv-

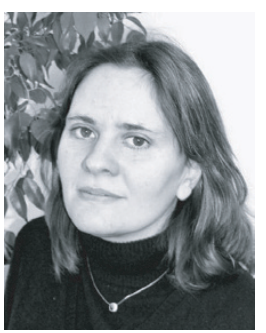
ing, in 1997, the Master's degree in telematics and multimedia applications from COREP, Politecnico di Torino, Italy. From 1997 to 2001, she worked as a Researcher in the Internet Services Division of Telecom Italia Lab (formerly CSELT), Italy, the research branch of Telecom Italia, where she matured experience in the field of Internet services, with a particular focus on electronic commerce and network security. Presently, she is a Ph.D. candidate at the International Graduate School of Information and Communication Technologies, University of Trento. Her main interests are video coding and transmission.

Francesco G. B. De Natale received the Laurea in electronic engineering in 1990, and the Ph.D. in telecommunications in 1994, both from the University of Genoa, Italy. After some years spent at the University of Cagliari as Assistant Professor, he joined the University of Trento, Italy, were he presently holds a Full Professorship in telecommunications. In Trento, he coordinates the B.S. and M.S. courses in telecommunications,

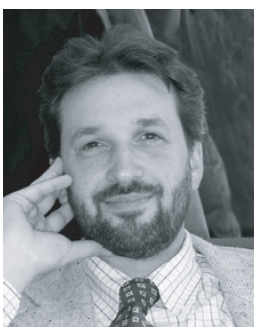
and is Deputy Head of the Department of ICT. He is also part of the Board of Directors of the National Consortium of Telecommunications (CNIT). The research interests of Professor De Natale are focused on image and signal processing, with particular attention to multimedia data compression, processing, and transmission. In this context, he leads the research activities of the Multimedia Communications Lab. He was General Cochair of the Packet Video Workshop in 2000 and Technical Program Cochair of the IEEE International Conference on Image Processing to be held in 2005. In 1998, he was corecipient of the IEEE Chester-Sall Best Paper Award. He is also Referee for several international journals in the field of signal processing and telecommunications, as well as Evaluator for the European Commission. Professor De Natale is a Senior Member of IEEE. 
Fabrizio Granelli was born in Genoa in 1972. He received the Laurea (M.S.) degree in electronic engineering from the University of Genoa, Italy, in 1997, with a thesis on video coding, awarded with the TELECOM Italy prize, and the Ph.D. degree in electronic engineering and computer science from the same university in 2001. Since 2000, he has been carrying on his teaching

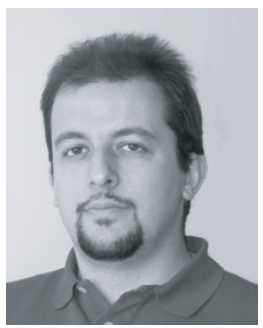
activity as Assistant Professor at the Depart-

ment of Information and Communication Technologies (DIT), the University of Trento, Italy, within the B.S. and M.S. degrees in telecommunications engineering. The research interests of Dr. Granelli are mainly focused on networking, with particular attention to network modeling and performance evaluation, access control, and next-generation telecommunication networks. He is an author of more than 30 refereed papers, published in international journals and conferences. Dr. Granelli is a Member of the IEEE Committee on "Communication Systems Integration and Modeling" (CSIM) and of the Technical Programme Committee of the "QoS and Performance Evaluation Symposium" of the International Conference on Communications (ICC 2003 and ICC 2004). He is Guest Editor of the special issue of the Journal on Special Topics in Mobile Networking and Applications (MONET) on "WLAN Optimization at the MAC and Network Levels." 